\title{
Prolegómenos a una teoría sobre la corrección de textos
}

\author{
NURIA GÓMEZ BELART \\ Universidad del Salvador \\ nuria.belart@usal.edu.ar
}

\section{Resumen}

Desde fines del siglo XIX, en Latinoamérica, se produjo un fenómeno bastante particular en el mercado editorial. Las diferentes actividades que se desarrollaban en este mercado iniciaron un lento proceso hacia la profesionalización de las tareas. Los escritores, los traductores, los editores, los periodistas y los publicistas ya no se ocupaban de otra actividad de modo paralelo, sino que invertían toda su energía en lo que, hasta el momento, se percibía como un pasatiempo y no como un trabajo que merecía ser reconocido como tal. Así surgieron nuevas profesiones, junto con espacios de educación formal, y, progresivamente, nacieron disciplinas científicas que le aportaban el sustento académico necesario a cada una de ellas.

En el caso particular de la corrección de textos, la profesionalización fue tardía y errática. Por esta razón, en este artículo se presentan los aportes de quienes impulsaron ese proceso de transformación durante la primera mitad del siglo xx: Manuel Benítez, Pelegrín Melús y Francisco Millá - dos correctores españoles que fueron tomados como referentes en la Argentina-, Eduardo Miragaya, José M. Rafols, Constancio C. Vigil, Raúl E. Lagomarsino y Carlos Pellegrini.

Finalmente, tomando como punto de partida el surgimiento de las primeras instituciones dedicadas a la formación académica de los correctores, se reflexiona sobre el papel del corrector en el proceso de creación y de publicación de un texto, las competencias que se requieren para llevar a cabo la tarea, el proceso de academización que experimenta la corrección como práctica profesional y la necesidad de avanzar en los estudios de la correctología, como disciplina lingüística emergente con un objeto de estudio delimitado y una base metodológica enmarcada en la pragmática y en la lingüística del texto.

\section{Palabras clave}

Corrección de textos; corrección de pruebas; correctología; lingüística aplicada. 


\title{
Prolegomena to a theory of copyediting
}

\begin{abstract}
Since the end of the 19th century, in Latin America, there has been a quite particular phenomenon in the publishing market. The different activities that were developed in this market began a slow process towards the professionalization of the tasks. Writers, translators, editors, journalists, and advertisers were no longer engaged in other activities in parallel, but instead invested all their energy in what, until now, was perceived as a hobby and not as a job that it deserved to be recognized as such. Thus, new professions arose along with spaces for formal education, and, progressively, scientific disciplines were born that provided the necessary academic support to each of them.

In the particular case of proofreading and copyediting, professionalization was late and erratic. This is why, this article presents the contributions of those who promoted this transformation process during the first half of the 20th century: Manuel Benítez, Pelegrín Melús and Francisco Millá -two Spanish copyeditors who were taken as references in Argentina-, Eduardo Miragaya, José M. Rafols, Constancio C. Vigil, Raúl E. Lagomarsino and Carlos Pellegrini.

Thus, taking as a starting point the emergence of the first institutions dedicated to the academic training of copyeditors, it reflects on the role of the copyeditor in the process of creating and publishing a text, the competencies that are required to carry out the task, the academicization process experienced by correction as a professional practice and the need to advance in the studies of Correctology, as an emerging linguistic discipline with a delimited object of study and a methodological base framed in Pragmatics and Text Linguistics.
\end{abstract}

\section{Keywords}

Copyediting; Proofreading; Correctology; Applied Linguistics.

Recibido el 18/05/2021

Aceptado el 06/06/2021 


\section{Introducción}

Por lo general, los críticos del mundo de la corrección ubican a los primeros correctores en tiempos de la invención de la imprenta. Sin embargo, la corrección existe de manera espontánea ${ }^{1}$ desde mucho antes de lo que se registra en los documentos porque es parte de la naturaleza humana el querer mejorar la creación propia o ajena. Pero no todo intento de mejora se basa en un método o se sustenta en alguna norma prefijada por la cultura. Por lo tanto, es necesario comprender que existe una diferencia fundamental entre las personas que señalan errores en un texto movidas por un impulso subjetivo y las personas con una «actitud» de corrección, es decir, una conducta consciente de mejorar un escrito de acuerdo con ciertos principios que justifiquen la enmienda, la reparación o la reescritura de un texto. A grandes rasgos, la historia de la corrección puede articularse en tres etapas: la primera se caracteriza por su modalidad espontánea o poco sistematizada sobre libros manuscritos, en la que había correctores cuyo oficio era revisar los textos, aunque no desarrollaban exclusivamente esta tarea; la segunda, ya organizada, en la que se cuenta con bibliografía sobre cómo llevar a cabo la tarea en las imprentas; la tercera, en la que la corrección se desarrolla no solo dentro, sino fuera de las editoriales, en un mundo digital y globalizado, donde el corrector vive una constante crisis, a causa de los avances tecnológicos y del continuo cambio en las competencias de los lectores.

Si bien este artículo podría comenzar por las primeras evidencias de esa «actitud correctora» en la Antigüedad, solamente se tendrán en consideración aquellos aportes que influyeron de manera más directa en el proceso de academización del oficio del corrector. Este proceso tuvo su origen en la Argentina, con el surgimiento de la Fundación Litterae, el primer ámbito de formación profesional de los correctores de textos; las primeras publicaciones especializadas, sobre todo, las obras de Alicia María Zorrilla, y la primera tesis sobre un tema específico de corrección de textos, en la que se plantea la necesidad de rever la percepción que se tiene sobre la práctica de corregir.

Durante el siglo xx, la corrección no fue un tema de interés para las universidades. En 1995, en la Argentina, unas pocas universidades privadas crearon los primeros espacios de formación académica y, al poco tiempo, se creó la cátedra de Corrección de Estilo en la Carrera de Edición de la Universidad de Buenos Aires. Existen varias instituciones que ofrecen títulos oficiales de corrector de textos, corrector de estilo o corrector literario, pero, en todas, la profesión es considerada una tecnicatura o un postítulo de especialización. Hasta ahora, salvo por un período muy breve tras el que se homologó con un título intermedio (en la Universidad del Salvador, en Buenos Aires), no existe la carrera de grado de Corrección de Textos.

A pesar de lo dicho, todo parece indicar que, en un futuro próximo, surgirán las carreras de grado, pues es una necesidad imperante en el ámbito de la corrección. Esto se debe a que la práctica profesional, como mínimo, exige las siguientes competencias:

\footnotetext{
${ }^{1}$ Se entiende aquí espontánea como 'aquella intervención que se produce por propia iniciativa sin formación académica específica para la tarea'. Se distingue del uso de intuitiva/o en la corrección, que se refiere a una capacidad de comprensión instantánea de los potenciales errores de un texto.
} 
el conocimiento de la gramática, de las normas particulares del registro adecuado al ámbito de publicación, de los contenidos de los que trata el documento y las estrategias textuales que permiten potenciar el escrito.

Con el objetivo de exponer los prolegómenos a una teoría sobre la corrección de textos, el presente trabajo se articula en dos instancias. Como todo proceso se desarrolla sobre la base de sus antecedentes inmediatos, en primera instancia, se presentará una síntesis diacrónica sobre los aportes de los correctores argentinos durante el siglo XX, que influyeron en la transformación de los correctores del mundo entero. En segunda instancia, partiendo de los avances en los estudios del lenguaje durante el siglo XX, se propone una reflexión sobre la corrección de textos desde una perspectiva fundamentada en las teorías lingüísticas que estudian el lenguaje en uso y que toman como unidad de análisis o bien el enunciado, o bien el texto, y abordan el lenguaje como un tipo de comportamiento para estudiarlo en relación con su contexto, es decir, con los principios extralingüísticos que regulan su uso en la comunicación.

\section{Los antecedentes inmediatos}

Desde fines del siglo XIX, en Latinoamérica, se produjo un fenómeno bastante particular en el mercado de las publicaciones eventuales y periódicas: las diferentes actividades que se desarrollaban en este mercado iniciaron un lento proceso hacia la profesionalización de las tareas. Los escritores, los traductores, los editores, los periodistas y los publicistas ya no se ocupaban de otra actividad de modo paralelo para subsistir económicamente, sino que invertían toda su energía en lo que, hasta el momento, se percibía como un pasatiempo y no como un trabajo que merecía ser reconocido como tal.

Así surgieron nuevas profesiones junto con los espacios correspondientes de educación formal, y, de manera progresiva, nacieron disciplinas científicas que le aportaban el sustento académico necesario a cada una de ellas. Sin embargo, el oficio de la corrección de textos no siguió el mismo rumbo que las especialidades afines. Hubo algunos intentos de elevar la figura del corrector, como el caso de Manuel Benítez, quien publicó Recuerdos de un corrector de imprenta (1925), pero fueron ejemplos aislados y no tuvieron un impacto directo en el mercado editorial.

Benítez fue una figura fantasma para la historia de la prensa y la literatura argentinas, uno de los tantos correctores anónimos de la primera mitad del siglo Xx. En su carta de advertencia explicaba la razón por la cual editaba sus memorias:

Camarada lector:

Evidenciar la importancia del papel que desempeña el corrector en el campo de las letras: tal es la finalidad que persiguen estas modestas páginas.

Me he valido para ello, como verás, de algunos recuerdos, en el transcurso de 30 años, corrigiendo, acá y acullá, pruebas y más pruebas para el libro y para la hoja diaria, las que me han condimentado (gracias a Dios, dicen... los que creen en él) el pan nuestro de cada día y, con él, el pan del espíritu, del que podemos proveernos, gratis y abundantemente, gracias, ahora sí, a los tipos que en aquella hora benditísima concibiera el obrero genial de Maguncia. 
Estos recuerdos traerán a tu memoria tantos y tantos otros con los que, por su analogía, estarán íntimamente eslabonados, recuerdos que nos hablan de satisfacciones e inquietudes, de injusticias y reparaciones, pruebas a que estamos fatalmente condenados en este valle de... errores, casi digo lágrimas, correctores y con correctores (Benítez, 1925: 11).

Benítez recordaba anécdotas referidas a errores tipográficos, opinaba sobre la imposibilidad de encontrar todos los errores de un texto y se quejaba de la poca preparación de los linotipistas, sobre todo, de su desconocimiento de las palabras y la poca curiosidad con que trabajaban los textos. Uno de los temas en los que insistía era el escaso interés que se le daba al oficio del corrector y el desprestigio en el que había caído la profesión.

Entre las quejas y los reclamos, comentaba que ni los tipógrafos ni los linotipistas entendían por qué el corrector devolvía las pruebas «tan sucias». Es decir, no comprendían por qué el corrector marcaba tantas comas y otras menudencias gramaticales. En cuanto a la paga, Benítez observaba que el corrector cobraba un dinero equivalente al de un peón ( 80 pesos), y que esa era una clara evidencia del poco reconocimiento que se le daba a los saberes que se necesitaban para corregir.

Como este, se publicaron varios textos referidos al oficio del corrector como un trabajo pleno de incógnitas que merecía reivindicarse. Según la mayoría de los autores de la época (Melús, Millá, Rafols, Pellegrini, Vigil, entre otros), nadie sabía con claridad cuáles eran las responsabilidades, ni los saberes que se requerían, ni la importancia de esta tarea, ni los métodos para llevar a cabo el trabajo con seriedad.

\subsection{Los aportes de Pelegrín Melús, con la colaboración de Millá}

Al menos para los correctores argentinos, el proceso del cambio se inició en 1937, en Barcelona, cuando se publicó El libro del corrector, escrito por Pelegrín Melús con la colaboración de Francisco Millá. En este manual se describían las tareas que desarrollaban los correctores de originales y de pruebas. Fue uno de los libros fundamentales para corregir. Era la fuente de consulta de toda persona que trabajara en la prensa, en las editoriales o en las imprentas.

A diferencia de los manuales que circulaban en la época, el de Melús componía la imagen del corrector desde su etimología y diferenciaba el corrector que enmendaba o enderezaba del que embellecía. Luego, describía las labores del corrector según la propia experiencia, sin los floreos academicistas de otros manuales.

Por primera vez, los correctores argentinos tuvieron un manual que los orientara de manera efectiva. En este manual, se especificaba que una de sus funciones principales era dirigir. Como representante inmediato del dueño del taller, debía asumir la dirección técnica, cuidar la organización interior, distribuir el trabajo, vigilar al personal, etcétera:

El corrector es, pudiéramos decir, el representante de la ciencia y del autor en el taller. Está sometido a la autoridad del regente como operario; pero en el ejercicio de su cargo goza de autoridad suprema y es el único juez y definidor 
competente en todas las cuestiones que caen bajo su jurisdicción (Melús, 1937: 9).

Como «el orden es garantía segura de una buena corrección», una de las responsabilidades del corrector era organizar las tareas para la publicación y mantener el equilibrio de las fases del proceso. Además, el corrector enmendaba, es decir, limpiaba los textos de cualquier desprolijidad, pero, por sobre todas las cosas, el corrector era «un colaborador del autor». El corrector perfeccionaba. El propio Melús explicaba que este concepto podía sonar redundante, aunque eso dependía de lo que se entendiera por perfección: el corrector debía tener la capacidad de potenciarla y darle «nuevos méritos». Pero toda modificación que se realizara tenía que estar aprobada por el autor. Ambos debían estar de acuerdo.

Con esta aclaración, Melús establecía una diferencia entre los correctores: algunos eran como obreros que detectaban erratas y las reparaban casi de manera mecánica, y otros eran personas reflexivas que buscaban embellecer el texto, además de detectar las erratas o cualquier otro tipo de error gráfico. Para este grupo de correctores, era necesaria una cultura vasta, formación literaria y gramatical, además de un profundo conocimiento de los circuitos de producción editorial y de la suficiencia técnica correspondiente:

En resumen, el corrector para poder desempeñar su cometido con la suficiencia y capacidad a que le obliga su cargo, ha de conocer a la perfección y prácticamente todos los recursos de la tipografía, ha de poseer una solidísima instrucción gramatical y ha de estar dotado de una cultura general bastante extensa que le permita, ayudado por su intuición y por su discreción (cualidades naturales que no deben faltar jamás y sobre las cuales se fundan todas las otras que hemos examinado), proceder con seguridad y con un criterio firme y sólido en el desempeño de su delicada misión (Melús, 1937: 20).

Melús detallaba los deberes del corrector sobre la base de una responsabilidad moral. Era claro que debía ocuparse de enmendar errores y evitar que aparecieran imperfecciones en el texto. De no hacerlo, el dueño de la imprenta era el perjudicado materialmente. Por eso, recomendaba que el último visto bueno fuera siempre el del autor, para descargar toda responsabilidad en él:

Casi resulta ocioso hablar de la responsabilidad del trabajo del corrector. [...] Claro que su responsabilidad no suele ser estrictamente material. Cuando por errores o imperfecciones hay que volver a hacer un trabajo, el responsable, es decir, el perjudicado materialmente, es el dueño de la imprenta. Todos los trabajos deberían ser revisados y autorizados por el autor antes de entrar en máquina, y esto descargaría al corrector de toda responsabilidad material.

La verdadera responsabilidad del corrector es puramente moral, y afecta a su conciencia, a su dignidad y a su prestigio; se deriva de la buena o mala organización de su trabajo, de su atención o descuido en la lectura, de su firmeza o debilidad en hacer cumplir las indicaciones señaladas en las pruebas, de la mayor o menor perfección de su método, de su constancia o negligencia en 
aumentar su cultura, etc., y se traduce en una serie de deberes más o menos imperiosos, o menos concretos, que trataremos de exponer y comentar lo más brevemente posible (Melús, 1937: 20-22).

También explicaba cómo debía ser el trato con el autor, que puede resumirse en la idea de respeto por la creación ajena, y con los tipógrafos; recomendaba evitar la soberbia o la vanidad por los saberes de que otros carecían. Si se comportaba de esa manera, lo único que conseguía el corrector era un mal clima laboral. Uno de los aspectos que podía deducirse de este manual es que la corrección de textos presupone la colaboración de varias personas: el autor, como primer interesado en que el texto se publique de la mejor manera posible; el corrector; el atendedor; el tipógrafo; el impresor, etcétera.

\subsection{Los aportes de Eduardo Miragaya}

Con el florecimiento de las publicaciones periódicas, las colecciones y la producción editorial, muchos de los correctores eran reconocidos escritores formados en letras o en periodismo, preocupados por la gramática y los avances de las distintas corrientes lingüísticas. Sin embargo, su fama provenía de sus creaciones y no de su trabajo de escritorio.

La primera mitad del siglo xx fue sumamente prolífica en cuanto a manuales y a otro tipo de herramientas para los correctores. Uno de los textos más conocidos en su tiempo fue el Diccionario de correcciones, de Eduardo Miragaya (1945), concebido, principalmente, para resolver las dudas y salvar las falencias de escritura en los periódicos. Este diccionario se compuso sobre la base de los errores que él mismo observaba con frecuencia en el diario argentino La Capital de Rosario, donde trabajaba como periodista y corrector. El trabajo de Miragaya adquirió tal relevancia en la prensa argentina que se publicó de manera independiente.

Uno de los aspectos más llamativos del diccionario era que el autor reconocía que no había tenido que esforzarse demasiado para encontrar errores y deslices en la escritura de los periodistas:

Como cazador de «gazapos» importa recalcar que, sin necesidad de ir a buscarlos, ellos vinieron solos, a la manera de las cacerías reales en que las piezas son llevadas hasta el cañón de la escopeta del monarca que no hace más que disparar y cobrarlas en abundancia. Es en el periodismo, cuyo lenguaje influye en el conocimiento del idioma, que tuvieron origen estos trabajos, y donde todo el esfuerzo se encamina a conservar en alto, sobre la marejada de incorrecciones, palabras y términos que dieron gloria a los escritores, de acuerdo, claro está, con el Diccionario. Y si un diario no puede hacer dejación del Diccionario que cuida como auxiliar de su propio prestigio, y a mayor prestigio corresponde[n] mayores preocupaciones por las formas de expresión y hasta de corrección académica. Se comprenderá bien que la indiferencia por estas cuestiones de lenguaje no se conciba; de aquí que su redacción se distinga por la claridad, de aquí, también, el que realice una labor pedagógica de primer orden (Miragaya, 1945: 11). 


\subsection{Los aportes de José M. Rafols y de Constancio C. Vigil}

José M. Rafols (1947) publicó Acento impreso. Notas para correctores, con un prólogo de Constancio C. Vigil, uno de los más grandes editores de la Argentina de su tiempo, quien comprendía que un manual para correctores debía focalizarse en la práctica:

Guiar a los que realizan su noble oficio es lo que el autor se propone con esta obra, eminentemente práctica, desprovista de didactismo, en [la] cual todo es útil para el corrector todavía bisoño. El lastre teórico que dice mucho y hace poco, que infla textos y programas, está mezclado con mucha parsimonia y sólo en la proporción necesaria para dar estructura al conocimiento (Vigil apud Rafols, 1947: 9).

El trabajo de Rafols se construyó sobre la base de la normativa del español establecida por la Real Academia Española y de algunos trabajos periodísticos «bien» escritos. En el mismo prólogo, Vigil reconocía la trayectoria de Rafols y le daba el lugar de autoridad en cuestiones de corrección. Es importante tener esto en cuenta porque, de alguna manera, el tener maestros en la Argentina estableció una posición lingüística respecto de la Real Academia Española:

[Las observaciones de Rafols] son de un hombre envejecido en el oficio, que recuerda que fué en su juventud maestro de escuela y que desea ahora ayudar a los nuevos correctores con los frutos de su larga experiencia y de su estudio. Noble empeño que prueba generosidad de corazón y merece la simpatía de los trabajadores del periodismo (Vigil apud Rafols, 1947: 10).

Rafols se focalizaba en las cuestiones de ortotipografía, sobre todo, en el uso de las tildes, pero también explicaba las bases de las reglas de la puntuación, en especial, del uso de la coma, a la que califica de «insignificancia gráfica» para explicar las causas de tantos errores que se cometen por su mal uso. Para las correcciones, seguía el criterio de autoridad, tanto para enmendar aquello que no se había escrito en un registro acorde con la lengua estándar como para reparar cuestiones de uso. Asimismo, desarrolló una suerte de apología del corrector e incluía el problema que muchos correctores tenían cuando debían revisar el escrito de un especialista o de un académico, el problema del ego profesional:

[...] entre la rigidez académica — tacañería intransigente, según algunosy la chabacanería cursi y efectista hay el rico venero que explotaron los Paladines de la lengua, y hoy, los Valera, los Estrada, los Sarmiento, los Palma, los Rojas, los Azorín, aunque no necesitamos tanto... pero tampoco tanto menos, como pretenden aquellos que aceptan que les enmendemos la plana y les cubramos las galeras de correcciones, jnosotros, los aprendices del idioma, los indigentes de la idea! Porque un corrector que reclama de los originales de un miembro de la 
Lengua o de la Historia, de un autor consagrado o de un maestro de la lengua, vaya al caso, no es un purista quisquilloso que se las echa de Aristarco, sino un obrero que, sencillamente, tiene que cumplir con su deber, de acuerdo con los principios establecidos, en defensa del buen sentido más elemental (Rafols, 1947: 110).

Pero, en favor del equilibrio, Rafols advertía a los correctores de los problemas de la sobrecorrección y de la facilidad con que se incurría en este vicio. También explicaba las consecuencias de asumir una posición suficiente en la que el corrector invadiera la obra ajena. Hacia el final del tratado, aportó algunos datos relativos al modus faciendi en la corrección de plomo, con los signos gráficos característicos, y establecía la diferencia con la corrección en caja, donde se podían mover los tipos sueltos.

\subsection{Los aportes de Raúl E. Lagomarsino}

En 1957, Raúl E. Lagomarsino, editor de la Sociedad de Bibliófilos Argentinos y Amigos del Arte, publicó Savia y follaje del libro, donde describió de manera práctica las normas y el procedimiento de trabajo para facilitar la tarea de «autores, traductores, correctores, linotipistas y tipógrafos». Ante todo, propuso el principio de la consistencia en los criterios: «Creemos que las equivocaciones que puedan originarse por la aplicación de un criterio uniforme siempre tendrán menos importancia que la anarquía derivada de distintas interpretaciones» (Lagomarsino, 1957: 14).

Para Lagomarsino, el texto era «materia viva», y, por lo tanto, los correctores debían buscar el modo de no «herir la expresión» ni «ahogarla por excesivo cuidado». Lo más importante de todo: debían tener «el mayor respeto hacia el autor». Pero también advertía a los correctores de no empeñarse u obsesionarse por reducir las expresiones, cuando había una razón estética elegida por el autor, o los vulgarismos utilizados en obras literarias o dramáticas, pues así modificaban el carácter de los personajes.

Después de establecer las bases del proceso de composición y de armado de un libro, el autor explicaba una serie de generalidades sobre la corrección en las que organizaba la tarea y describía, paso por paso, el procedimiento. «En la indicación de las correcciones debe cuidarse de observar sencillez, orden y claridad» (Lagomarsino, 1957: 118). Como contaba con la experiencia de haber trabajado en los circuitos editoriales, Lagomarsino incluyó en el manual recomendaciones fundamentales para que el trabajo en equipo se desarrollara con fluidez y advertía sobre los problemas que podían suscitarse por no conocer las tareas que desarrollaban los demás integrantes de la editorial, sobre todo, el tipógrafo y el linotipista.

\subsection{La tesis de Carlos Pellegrini}

El trabajo en equipo y el puntillismo que se esperaba de la producción de un libro le dieron el fundamento a Carlos Pellegrini para afirmar, en La industria gráfica argentina (1948), que se trataba de una actividad artesanal: 
No invalida esa clasificación el hecho de que algunos productos salidos de las imprentas, tales como libros en blanco o impresos, tarjetas postales, etc., conserven características comunes con la venta de artículos de procedencia fabril.

En verdad son pocas las Imprentas que por su cuenta y bajo su propia dirección ejecutan esos trabajos, la más de las veces existe un intermediario, para quien en verdad se ha trabajado, de acuerdo con sus directivas. Además, si la imprenta y la editorial están en manos de un solo empresario no por ello dejan de ser dos las actividades que éste desarrolla, y ambas con características propias (Pellegrini, 1948: 25-26).

Enmarcada en una actividad artesanal o no fabril, Pellegrini establecía una serie de clasificaciones sobre los procesos y el perfil de los trabajadores que desarrollaban las actividades. Para el Estado, en 1939, la industria gráfica era catalogada como: «Imprenta, publicaciones y análogos», y se la subdividía en los siguientes rubros:

1. Imprentas, incluso litografía y talleres de encuadernación.

2. Diarios, periódicos y revistas.

3. Industrias anexas de las artes gráficas (estereotipia, fotograbado, fundición de tipos, composición, etc.) no efectuados en imprentas o talleres de diarios, periódicos o revistas (Pellegrini, 1948: 28-29).

De esta catalogación, se deducía que los correctores formaban parte de un «etc.» abreviado, y que, por lo tanto, no eran considerados parte del circuito editorial o de la industria gráfica. Más sorprendente aún es cuando, en su tesis, Pellegrini observó que la Argentina era el segundo país productor de la industria editorial en el continente (Estados Unidos era el primero).

En la tesis, se nombraban las asociaciones vinculadas con la industria gráfica, se describían las características de las imprentas de acuerdo con la cantidad de personal con que contaban, y se aportaban un sinnúmero de datos estadísticos sobre la producción editorial, pero solo se nombraba a los correctores una vez, cuando se indicaba el monto que ganaban por hora trabajada. Para ese entonces, los correctores eran considerados oficiales, según el régimen de tarifas de la industria editorial. Esto quiere decir que debían ser egresados de las escuelas de aprendizaje y de orientación profesional que acreditaban fehacientemente su idoneidad. En «Corrección», figuraban los oficiales de primera corrección, con conocimientos gramaticales y tipográficos —en otras palabras, personas con mucha experiencia o con larga trayectoria editorial y que, además, supieran gramática一, y los oficiales de segunda corrección, que implicaba lectura o confrontación - personas con experiencia en el circuito editorial, pero que no necesariamente sabían gramática-. El oficial de primera cobraba entonces, según los registros oficiales, 2,88 \$ por hora trabajada, mientras que el oficial de segunda, 2,50 \$.

Es importante tener en cuenta que un peón de una estereotipia cobraba 2,27 \$ y que el sueldo de un oficial de primera era bastante menor que el de un maquinista. Por lo tanto, el trabajo de corrector, desde la perspectiva salarial, era equivalente a la de un obrero de formación mínima; pero los oficiales debían cumplir con un nivel de formación superior para poder desarrollar la tarea. 


\subsection{Una reflexión sobre los antecedentes}

Desde una perspectiva diacrónica, se puede observar que el desarrollo profesional de los correctores no solo fue tardío, sino que estuvo plagado de obstáculos. Al menos en la Argentina, resultaba incomprensible la razón de ser de esta clase de profesionales en el contexto editorial. Pocos podían dar cuenta de las funciones que desarrollaban en el circuito de la producción de publicaciones; pocos percibían la utilidad de contar con correctores en las imprentas o en las casas editoriales.

Además, como ralentizaban el proceso por su exceso de puntillismo y los pedidos de remaquetación, los correctores se ganaron muchos enemigos en los equipos de trabajo que integraban. En este contexto, la figura del corrector cayó en desgracia, circunstancia que se agravó con los avances tecnológicos y los primeros sistemas informáticos para la producción editorial. El corrector se convirtió en una molestia para la mayoría.

Con el vértigo de los avances tecnológicos, se produjo una nueva transformación del perfil del escritor y del editor. Se profesionalizaron las tareas de escritura, excepto, el oficio del corrector, que inició un lento descenso rumbo a una potencial extinción. Es llamativo el hecho de que, así como, en Europa, había conciencia histórica sobre el oficio, y se reconocían los nombres de los correctores destacados, en América, prácticamente, no se conocen los nombres de los primeros correctores (Gómez Belart, 2019). En la Argentina, por ejemplo, se sabe que el primer corrector fue un tal don Antonio Ortiz, al que hacen referencia unos pocos documentos y que, por lo tanto, estaba perdido en el olvido. Los correctores argentinos desarrollaron algunos pocos manuales de estilo, siempre orientados al trabajo interno de las publicaciones. En consecuencia, pasaron a la historia como figuras anónimas, salvo, quizá Constancio Vigil, quien fue el creador de muchas de las publicaciones periódicas del siglo Xx.

Con el inicio de la era digital, se produjo una revolución en la escritura. Publicar se convirtió en algo asequible para todo el mundo, sin que importara la formación, el prestigio o las competencias para escribir, y, paralelamente, con la invención de los autocorrectores digitales, se instaló la creencia de que los correctores ya no eran necesarios.

Afortunadamente, a partir de la década de los noventa, los correctores encontraron el modo de adaptarse y evolucionar de acuerdo con las nuevas exigencias y, sobre la base de los avances en los estudios del lenguaje, lograron redefinirse en un nuevo perfil: una figura de mediación entre el autor, el lector y el texto, cuya tarea es lograr la efectividad en las comunicaciones. Como la mayoría de los correctores incorporó erráticamente los conocimientos para esta nueva forma de ser profesional, se crearon espacios de entrenamiento y especialización, y, posteriormente, de encuentro y debate.

Hacia fines del siglo Xx, se inició un camino hacia la profesionalización que supuso una reinvención del perfil del trabajador. En consecuencia, se redefinió el vínculo entre el autor, el texto, el corrector, el ámbito de publicación y el lector.

En Buenos Aires, la primera institución orientada a formar correctores fue la Fundación Instituto Superior del Estudios Lingüísticos y Literarios Litterae, creada y 
dirigida por Alicia María Zorrilla. Desde su inicio, en 1988, buscó no solo formar correctores mediante clases y cursos de especialización, sino que lanzó revistas, editó manuales de todo tipo, en especial, con una sólida base normativa. A partir de 1992, se llevaron a cabo las Jornadas sobre Normativa del Idioma Español, que fueron la semilla de los Congresos Internacionales de Corrección de Textos en Español (CICTE), cuyo primer encuentro tuvo lugar en Buenos Aires, en 2011, y que llevó el diálogo entre profesionales a un debate internacional.

Si se analiza la evolución del perfil profesional del corrector, se puede observar que, durante los últimos veinte años, se produjo un viraje alentador rumbo a la academización, y, gracias a los CICTE y a la globalización, este perfil profesional tuvo un crecimiento vertiginoso no solo en cuanto a su formación, sino en cuanto a su inserción en el mercado. Es más, surgieron los primeros estudios formales sobre la práctica de corregir y, en noviembre de 2019, se defendió la primera tesis doctoral que estudiaba la corrección de textos como disciplina lingüística. Con ella, finalmente, nació la correctología 2 .

\section{Una ciencia de la corrección de textos}

Así como otros especialistas han sentado las bases para el desarrollo de otras disciplinas lingüísticas, la corrección necesitaba iniciar su camino disciplinar a partir de la reflexión sobre la propia práctica. A lo largo de los últimos años, principalmente como resultado de los avances tecnológicos, se produjo una transformación del perfil del corrector. Tal circunstancia conllevó una reflexión más profunda respecto de su papel en el proceso de creación y publicación de un texto, las competencias que se requieren para llevar a cabo la tarea, cuáles son las posibilidades de participación en ese proceso, los métodos que favorecen la realización de la tarea, la finalidad última de la corrección.

\subsection{Reflexiones sobre el problema del objeto de estudio}

Como en todas las áreas del saber, es necesario considerar las condiciones necesarias y adecuadas para la formulación de una teoría lingüística que establezca un método para comprender su objeto. Por lo tanto, es necesario definir el objeto de estudio de la correctología: si se parte de una definición general, como la disciplina que se ocupa del estudio de la corrección de textos, se vuelve evidente que uno de los mayores obstáculos por afrontar es la falta de precisión sobre el criterio para conceptualizar el objeto. Dicho de un modo más simple, hay que establecer qué se entiende por corrección.

Hasta el momento, el proceso de corrección como fenómeno lingüístico se ha estudiado con profundidad en muy pocas circunstancias. Teniendo en cuenta las sucesivas transformaciones que se dieron en el ámbito de la escritura y de la lectura, en

\footnotetext{
2 En noviembre de 2019, la autora de este artículo presentó su tesis: La corrección de textos. Un estudio funcional del vínculo entre el autor y el corrector. Su directora fue la Dra. Alicia María Zorrilla y el jurado estuvo integrado por la Dra. Beatriz Curia, la Dra. Hilda Albano y el Dr. José Luis Moure.
} 
cuanto a los medios de publicación, al perfil del escritor y, en consecuencia, a los textos propiamente dichos, es fundamental realizar una teorización sobre este proceso.

Si bien existe la creencia de que los correctores se dedicaban solamente a revisar los textos hasta un nivel oracional o a observar cuestiones puramente ortotipográficas, a partir de los primeros estudios en el área de la correctología, se demuestra que, desde el surgimiento de la práctica, los correctores revisan mucho más que la sintaxis, la precisión semántica o la adecuación léxica. Por esta razón, es imprescindible analizar el estado de la cuestión hasta el momento y reconsiderar como se lleva a cabo este proceso.

Como se dijo, apenas existen trabajos académicos formales que aborden esta problemática en los términos planteados, así como tampoco hay información suficiente sobre qué se debe corregir y qué no, cuál es el límite entre el trabajo del corrector y el del autor, cuáles son los conflictos que se generan en el proceso de una corrección, etcétera. Tampoco existe material alguno sobre los parámetros o los fundamentos lingüísticos que sirven de sustento para la corrección de textos, salvo los documentos publicados durante los últimos diez años en los Congresos Internacionales de Corrección de Textos en Español (CICTE), los manuales y las publicaciones orientadas o bien a las cuestiones normativas, o bien a las experiencias personales de algunos correctores. Estos materiales son sumamente útiles para los correctores porque resuelven las inquietudes propias de la práctica, pero no son publicaciones científicas.

A pesar de lo dicho, en este oficio es común encontrar personas que asumen la tarea porque creen que basta contar con el dominio de una buena ortografía. Sin embargo, durante las últimas décadas, los estudios sobre corrección se concentraron, entre otros aspectos, en el tipo de competencias necesarias para desarrollar la tarea. Como señala Zorrilla (2011), se trata de una práctica que requiere de cuidados especiales, y no todas las personas están preparadas para desarrollar esa actividad.

Además de la falta de certeza sobre las competencias que se requieren para desarrollar la tarea y de los problemas estrictamente metodológicos, los correctores actuales debieron adaptarse a una nueva situación editorial que los condujo a cuestionarse la identidad y la incongruencia entre los requerimientos y el prestigio que se les reconoce. El corrector era percibido como un ser antipático, cerrado y autoritario. Sin embargo, la realidad actual de los correctores está muy lejos de esa representación.

Corregir es una actividad compleja que requiere de numerosas competencias: aparte de la enciclopédica, se espera que el corrector sea competente en términos lingüísticos no solo desde el punto de vista formal (el conocimiento de la gramática y de la normativa, por ejemplo), sino también desde el punto de vista sistémico-funcional y cognitivo; esto último implica conocer el entorno sociolingüístico, detectar las expectativas del lector potencial o conocer las características del soporte en el que se publicará la obra, pero, además, comprender cómo se genera el pensamiento en el escritor que compone determinada oración con una arquitectura concreta o que apela a una metáfora, por ejemplo, para ilustrar un concepto. También exige competencias estratégicas y editoriales, que le permitirán decidir cuáles son las mejores formas para enmendar el texto y en qué momento del proceso debe realizarse esa tarea. Asimismo, es conveniente contar con las competencias necesarias para una escritura clara - 0 bella- y adecuada, porque esto le facilitará la toma de decisiones sobre cierto tipo de 
errores, y con la competencia visual, es decir, el ojo entrenado para detectar errores, anomalías, inconsistencias, oscuridades, etcétera.

Hoy se sabe que existen especialidades en el mundo de la corrección, aunque solo a fines del siglo xx, comenzó a especificarse su campo de trabajo. Es probable que este fenómeno se explique por la profesionalización tardía de la escritura y de las especialidades afines, con la consecuente transformación del perfil de los escritores no solo en cuanto a su dedicación y su método, sino en la accesibilidad para publicar, condición que se acentuó con el surgimiento de las computadoras personales y de la internet.

Partiendo de la metodología propia del corrector y del vínculo que supone trabajar con autores que componen determinados tipos de textos, con el surgimiento de la correctología se dio el paso necesario para que el oficio de corregir sea entendido como una disciplina lingüística sustentada en bases formales y funcionales, y que reciba el reconocimiento académico que merece, de la misma manera en que se reconocen la traducción, los estudios sobre el libro, la edición o la escritura académica.

La correctología es la disciplina que estudia los procesos de corrección con el fin de describir la interacción entre un sujeto productor (un autor), un sujeto receptor (un lector), un sujeto corrector, el texto (plasmado en un soporte determinado) y la situación comunicativa en la que estos participantes se vinculan (el contexto).

\subsection{Reflexiones sobre el problema de la base metodológica}

\subsubsection{Una disciplina enmarcada en la pragmática}

La teoría exige una base metodológica para alcanzar el propósito de describir los procesos de corrección. Esta descripción debe cumplir tres condiciones: ser autoconsistente - es decir, que no presente contradicciones internas-, exhaustiva y lo más simple posible.

Cada proceso de corrección es único, puesto que conserva cierta impronta artesanal, es decir, no seriada ni automatizada. Por esa razón, conviene tomar como sustento teórico un marco en el que se pueda analizar cada situación comunicativa de manera individual, pero que, a su vez, permita la reflexión sobre otros procesos de corrección, ya sean similares o contrastivos.

En este sentido, desarrollar un estudio funcional sobre la corrección de textos resulta sumamente productivo. En otras palabras, es fundamental estudiar el papel que cumple quien interviene entre el autor y el lector, en una situación comunicativa mediada por una serie de principios que regulan el uso del lenguaje en el proceso de la producción textual.

Como la pragmática es una disciplina lingüística que estudia el lenguaje asociado a su uso y a la acción en que ocurre, los enunciados son tomados por unidades de análisis, lo que significa que también se tienen en consideración el contexto y las proformas, el problema de la subjetividad y de la objetividad, los actos de habla, las condiciones de verdad y la presuposición, las inferencias, las implicaturas y las explicaturas, así como las representaciones enunciativas. Entonces, siguiendo esta perspectiva, son fundamentales para el estudio de la pragmática los factores extralingüísticos que 
determinan el uso del lenguaje, es decir, aquellos factores de los que no pueden establecerse referencias en un estudio puramente gramatical, como la identidad del emisor y del destinatario, la intención con la que se da ese mensaje, el contexto verbal, la manifestación, la situación en que se da el diálogo o el conocimiento del mundo que tienen los participantes.

La importancia de un análisis desde esta perspectiva metodológica reside en que la realidad del trabajo del corrector implica una intervención dentro de lo que, tradicionalmente, se analiza como esquema de comunicación. Tomar el modelo de comunicación de Roman Jakobson (1963) o el de Catherine Kerbrat-Orecchioni (1990) puede ser productivo para una corrección de carácter ortotipográfico o, hasta cierto punto, una corrección gramatical, pero, cuando se trata de un trabajo integral, estos modelos no funcionan como base teórica porque no tienen en cuenta la textualidad de un escrito o el conjunto de expectativas que tiene el lector cuando recibe un texto que responde a un formato determinado.

Pensar que las lenguas son solo códigos que se utilizan para transmitir un mensaje evidencia una perspectiva muy simplificada de lo que ocurre en la comunicación. El entorno demuestra que las lenguas no solo son codificaciones, sino que hay una cultura subyacente y un conjunto de exigencias y de expectativas que deben satisfacerse para que la información llegue a destino con éxito. Es más, si las personas se expresaran en sentido lineal, se limitarían las formas de expresar los afectos, sería imposible hablar de aquello que no se ciñe a una definición o a una palabra y se restringirían las posibilidades creativas para hablar de las propias creencias, del amor o de la muerte.

En una oración, pueden observarse dos niveles de interpretación. En el primero, se detecta que las palabras expresan el significado literal del enunciado, objeto de la semántica. Se lo suele llamar significado convencional, porque parte del sentido más frecuente que una comunidad asigna a una expresión determinada. En el segundo, hay un significado distinto del que se percibe superficialmente; es intencional y depende no solo del acto de emisión en que se produzca, sino también del contexto. Cada uno de estos significados se manifiesta en combinación con otras unidades lingüísticas que permiten que el interlocutor comprenda que el emisor no está haciendo referencia al significado compartido por convención, sino que lo está utilizando con un valor semántico diferente.

Cuando Sperber y Wilson desarrollan la teoría de la relevancia (1986), observan una diferencia entre uso descriptivo y uso interpretativo, y esta consideración es fundamental para el análisis de un corrector. Los autores explican, por una parte, que un enunciado es una descripción cuando su contenido proposicional refleja un estado de cosas o es utilizado de una forma descriptiva en que la información de dicho enunciado coincide en su mayor parte con la intención de lo que se quiere informar. Por ejemplo, cuando alguien dice «Andá a ver si llueve», porque está por salir y no sabe si llevar paraguas o no. Por otra parte, Sperber y Wilson observan que está usado de manera interpretativa cuando representa otra forma proposicional, a la que se asemeja en algún aspecto. En otras palabras, el contenido de lo dicho no concuerda con el uso que se da a ese enunciado y, por lo tanto, exige, por parte del interlocutor, una reflexión que permita comprender la verdadera intención del mensaje, como cuando se dice «Andá a ver si 
llueve», porque es una de las tantas expresiones coloquiales que existen en la Argentina para pedirle al oyente que se retire o para hacerle saber que está molestando.

La noción de uso interpretativo no es una simple variante terminológica de nociones como la de discurso referido o la de cita, definidas por Reyes (1990), sino que posee propiedades que la apartan de estos conceptos, pues la representación construida por un enunciado usado interpretativamente es siempre selectiva. Esto quiere decir que puede compartir las propiedades de un enunciado de tipo descriptivo, pero siempre hay alguna transgresión que permite al interlocutor comprender que no se trata de un mensaje lineal. Siempre que se reproduce o se recrea una cita, hay una intención en la réplica que enriquece el mensaje y lo desvía de su objetivo inicial.

Por ejemplo, cuando Esteban Echeverría asigna una cita mínima en los epígrafes a cada canto de La cautiva (1837), las palabras de los autores se resignifican y, en el poema, funcionan como focalizadores del contenido; pero, además, se enriquecen con la información que el lector tiene de los autores y de las obras citadas. En el caso del primer canto, el epígrafe es el siguiente: «Ils vont. L'espace est grand» [Ellos van. El espacio es grande] (Echeverría, 2010: 35). El epígrafe proviene de «Mazeppa», poema xxxiv de Les Orientales, de Victor Hugo (1829). Como señala Beatriz Curia, con el verso de Hugo, Echeverría presenta la inmensidad del desierto vacío, pero también incluye la desenfrenada cabalgata de los indios después del malón. De todas maneras, el epígrafe no se focaliza solamente en esta parte de la obra: «toda La cautiva presenta el desierto en su vastedad solitaria y el peregrinaje de los protagonistas a través de ella» (Curia, 2010: 1).

Como se observa en el ejemplo, leer una obra no solo implica una decodificación gramatical o normativa, sino que es necesario comprender que los enunciados, las obras, exigen un saber que excede los límites de lo que habitualmente se entiende por lenguaje. Exigen comprender la cultura en que ese enunciado se expresa. Si bien no hay estudios académicos que aporten datos para el trabajo del corrector, este concepto no es nuevo. Ángel Rosenblat (1967) explicaba su teoría de las variaciones lingüísticas a través del siguiente ejemplo: si en alguna calle de la ciudad de México se observa un cartel en el que se indique: «Prohibido a los materialistas detenerse en lo absoluto», una persona ajena al contexto donde se lleva a cabo el enunciado podría entender que, en las señales de tránsito mexicanas, se exponen dilemas filosóficos. Sin embargo, en algunas regiones de México se entiende por «materialista» una persona que transporta materiales para la construcción en un camión; por lo tanto, «en lo absoluto» se refiere a una prohibición tajante, y no al antónimo de «relativo».

Aunque, por lo general, la pragmática estudia enunciados en los que el hablante es quien tiene la intención de referirse a algo distinto del significado literal, también es frecuente que la sobreinterpretación del significado del hablante conduzca a una discusión entre los participantes de la conversación. Por ejemplo, si, con la intención de halagar, un muchacho le dice a su novia: «Qué linda que estás hoy», lo más probable es que ella se ofenda y le responda: «Yo soy linda, mi amor» o «¿No estoy linda otros días?».

En la comunicación, la lengua es un código para transmitir datos, pero esta idea no es suficiente para describir lo que ocurre en una conversación, o cuando un lector se acerca a una obra. No hay una correspondencia unívoca entre la imagen acústica (o escrita) y el concepto al que se refiere. Pero, a pesar de ello, no constituye 
necesariamente un obstáculo para la comunicación. Como sostiene Escandell Vidal (1996), resultan naturales expresiones como «leer entre líneas», «leer en contexto» o las reformulaciones.

En consecuencia, el corrector señala no solo los posibles errores ortotipográficos o los errores normativos, sino que tiene que interpretar el papel de lector y verificar si los términos son claros, si el léxico es inadecuado, si el discurso es confuso o si se brinda la información necesaria para comprender el contenido, de acuerdo con la enciclopedia (los saberes previos) del público al que se dirige ese texto.

Como se ha señalado, existe una distancia entre lo que literalmente se dice y lo que el hablante quiso decir. En el pasaje de la estructura profunda a la estructura que se manifiesta en superficie, se producen mecanismos de adecuación de las secuencias gramaticales al contexto del contenido y de la situación comunicativa, y se observa la asignación de referentes para la comprensión y la agilidad del mensaje.

Las expresiones que utiliza un autor en un texto no siempre guardan relación directa con el significado literal de las palabras que las componen, sino que dependen de datos que surgen del contexto de la comunicación. Por eso, se afirma que hay una parte del significado transmitido que solo puede ser comprendida en función de las circunstancias en que se enmarca el texto.

Por esta razón, es conveniente que el corrector se sustente en teorías generales del lenguaje que puedan explicar cómo es posible que lo que se quiere decir y lo dicho no coincidan y que, aun así, el interlocutor comprenda el mensaje. Conociendo aquellos problemas que constituyen el centro de interés de la pragmática, el corrector tiene un mayor número de herramientas para orientar al autor sobre aquellos aspectos de su escrito que son inadecuados o poco efectivos. Además, puede señalar cuestiones sobre la falta de legibilidad de un escrito de acuerdo con el lector potencial que el autor haya imaginado.

La perspectiva pragmática aporta muchas ventajas al trabajo del corrector: complementa las consideraciones gramaticales, a la vez que «contribuye no solo a ofrecer una visión más precisa de la compleja realidad lingüística, sino, sobre todo, a simplificar notablemente la descripción del nivel estructural» (Escandell Vidal, 1996: 23).

\subsubsection{Una disciplina enmarcada en la lingüística del texto}

Como el análisis de la situación lingüística real queda reducido a puras abstracciones inconexas si se estudian solo oraciones, en la actualidad, se abordan los estudios de la lengua en el encuentro comunicativo real de dos interlocutores. Esta nueva perspectiva, la llamada lingüística del texto, junto con la pragmática, supera el límite tradicional de la oración como unidad central y asume el texto como su objeto principal de estudio.

En un comienzo, lingüistas como Meter Hartmann se propusieron el objetivo de describir el discurso en términos estructurales. El grupo de Constanza (Petöfi, Kummer, Ballmer) se centró en la coherencia del discurso; en los aspectos pragmáticos en el uso de la lengua; en las relaciones de la actuación lingüística con una teoría general de la acción; en la incorporación de categorías de la lógica, como la noción de mundo posible 
para explicar la coherencia semántica del discurso. A partir de estos estudios, se extendió el campo de análisis, hasta que, en 1980, Van Dijk publicó Texto y contexto. Semántica y pragmática del discurso, un esbozo sistematizado de la lingüística del texto. Luego, Lozano, entre otros lingüistas, integró la semiótica en los estudios de la lingüística textual. Entonces, se ensamblaron las ideas de Van Dijk y de Eco, así como las investigaciones de la pragmática lingüística y de la teoría del discurso de Greimàs.

Llegado este punto, hubo que repensar el concepto de texto, puesto que cada definición aportada por un lingüista resultaba incompleta o insatisfactoria para los demás. Por ejemplo, para Umberto Eco (1979), el texto es un artificio sintácticosemántico-pragmático cuya interpretación está prevista en su propio proyecto generativo. Como tal, es un mecanismo perezoso que necesita de la cooperación interpretativa del lector. En El método formal en crítica literaria (1928), el texto, tanto en su forma escrita como oral, es considerado por Mijaíl Bajtin la base de todas las disciplinas $\mathrm{y}$, en general, de todo el pensamiento teológico y filosófico desde sus orígenes. Como el texto es una realidad inmediata y donde no hay texto no hay tampoco materia de investigación y de pensamiento, el hecho de considerarlo objeto permite la convergencia de disciplinas. Por su parte, Lotman (1972) y la escuela de Tartu conciben el texto como conjunto sígnico coherente, como formación semiótica singular, cerrada en sí, dotada de un significado y de una función íntegra y no descomponible.

Si bien las diversas tendencias en la lingüística textual hacen que no exista una definición que satisfaga a todos, Enrique Bernárdez, en la Introducción a la lingüística textual (1982), extrae el factor común a las distintas definiciones y determina que hay que considerar distintas variables cuando se define un texto: su carácter comunicativo, porque se trata de una actividad; su carácter pragmático, pues contiene la intención del hablante y se da en un contexto; y su carácter estructural, ya que se construye sobre la base de reglas propias del nivel textual.

Como el texto es un enunciado, constituye una unidad lingüística comunicativa, producto de la actividad verbal humana, que posee siempre carácter social; está caracterizado tanto por su cierre semántico comunicativo como por su coherencia profunda y superficial, debida a la intención del hablante de crear un discurso íntegro, y a su estructuración mediante dos conjuntos de reglas: las propias del nivel textual y las del sistema de la lengua.

En relación con un escrito, el contexto es la reconstrucción teórica de una serie de rasgos de una situación comunicativa, es decir, aquellos rasgos que integran las condiciones que hacen que los enunciados den resultados como actos de habla. La relación entre texto y contexto se da en ambos sentidos: algunos rasgos textuales permiten expresar aspectos de la situación comunicativa - como en el caso de los deícticos-; pero también la estructura del contexto define en gran medida qué rasgos deben disponer los textos para ser aceptables - por ejemplo, la estructura de una monografía tiene sentido en un contexto académico, pero, probablemente no lo tenga en el ámbito publicitario-.

Las relaciones semánticas y las pragmáticas entre texto y contexto se articulan de acuerdo con la intención comunicativa, el vínculo entre el autor y el lector, y los supuestos en juego. Que el texto y el contexto estén ligados sistemáticamente entre sí resulta de las relaciones entre el contenido y las condiciones para el logro de los actos 
de habla. Un manual para niños de seis años es concebido para que un niño interactúe con él, adquiera algún saber o potencie sus competencias respecto de un tema, y se presupone que el lector tendrá determinados conocimientos, carecerá de otros, estará en la etapa de desarrollar sus habilidades de lectura y comprensión, etcétera. Si una persona adulta leyera este manual, la situación comunicativa se redefine, y, en consecuencia, aunque no pierde su contenido en cuanto a la semántica, se reinterpreta la intención del autor y se produce un viraje en la utilidad o en la finalidad del libro.

Un concepto fundamental para comprender lo que ocurre con los rasgos textuales es el que aportaron Van Dijk y Kintsch (2006): el modelo situacional, una noción que también fue utilizada, aunque de manera diferente, por el psicolingüista Johnson-Laird en su libro Modelos mentales (1983). «El punto central de tal noción es que los usuarios de la lengua no construyen simplemente la representación (semántica) del texto en su memoria episódica, sino, además, una representación acerca del acontecimiento o de la situación del texto» (Van Dijk y Kintsch, 2006: 9). Esta idea de modelo dio fundamentos a la teoría de la coherencia referencial: un texto es coherente cuando condice con un modelo. En otras palabras, si la gente puede construir un modelo plausible para una secuencia o un texto completo, entonces el texto es subjetivamente coherente. También resuelve el problema de la referencia extralingüística y del análisis del discurso, porque el contenido refiere siempre a la reconstrucción del mundo intersubjetivo, o de una situación en el mundo, de acuerdo con sus modelos mentales. De esta manera, los especialistas resolvieron el problema de la coherencia referencial, que había sido definida de acuerdo con los modelos formales o de los mundos posibles.

Con la idea de modelo, las macroestructuras de los textos se pueden explicar de manera más precisa: no son visibles o expresadas directamente en el discurso en sí mismo, pero la gente sabe cuáles son los temas generales representados en su modelo mental de un acontecimiento. Pero, además, los modelos explican por qué la gente, a veces, recuerda un texto o la información que nunca fue expresada explícitamente en el texto original:

Así, si asumimos que la gente durante el proceso de comprensión también construye un modelo del acontecimiento, y si mucha de la información de tal modelo se puede derivar de un conocimiento más general, sociocultural, entonces estas «memorias falsas» pueden explicarse siguiendo el contenido del modelo construido para un texto. Es decir, lo que la gente recuerda de un texto no es tanto su significado, sino más bien el modelo subjetivo que ellos construyen sobre el acontecimiento del que trata el texto. Esto es por supuesto trivial cuando nos damos cuenta de que la mayoría de los lectores están interesados no tanto en el significado abstracto de un texto, sino en la información acerca de la realidad (Van Dijk, 2006: 11).

De lo dicho se puede afirmar que entender un documento presupone que la gente es capaz de construir un modelo mental del texto, y que lo mismo sucede en el momento de producirlo: este es el punto de partida de cualquier composición escrita, y también es el punto de partida para la corrección.

Los modelos pueden ser semánticos o contextuales (también se llaman pragmáticos), puesto que, en el contenido, se representan las características del hablante, de los demás participantes del discurso, del tiempo y del lugar, de las 
características sociales, de las relaciones entre los participantes, de los objetivos, los propósitos y los fines.

Estos modelos son la base mental de los actos del discurso dependientes del contexto, del estilo y de la retórica. En otras palabras, «controlan la manera en la que la información de los modelos de los acontecimientos se selecciona y se expresa eventualmente en el discurso» (Van Dijk, 2006: 13).

Por su parte, Swales (1990: 58) propone trabajar desde la perspectiva del género; en este aspecto, se concentra en el objetivo comunicativo y en las convenciones que comparte la comunidad discursiva que lo utiliza, según el contenido, la estructura, el estilo y la audiencia. La naturaleza esquemática del género facilita la comprensión por el conocimiento previo que los hablantes suelen tener de las convenciones que conforman cada texto, pero los estudios realizados sobre conocimiento previo y género, según Swales, demuestran que la comprensión lectora se afirma como consecuencia de la enseñanza de la estructura textual en sí misma. Un lector que no está formado o que nunca realizó el ejercicio de reconocer estructuras, aunque sea de modo espontáneo, probablemente tendrá dificultades para comprender el texto que se le presente (Swales, 1990: 89).

Definido en términos generales el concepto de texto y la estructura que lo compone, es fundamental para el corrector conocer las propiedades de la textualidad planteadas por De Beaugrande y Dressler (1997), que le permiten establecer criterios de trabajo en función de si un escrito cumple con los principios que regulan la textualidad en cuanto a la coherencia, la cohesión y la intención, además de establecer el grado que se exige al lector en cuanto al contenido por el tipo de intertextualidades que lo componen y la pertinencia en cuanto a la situacionalidad, la aceptabilidad y el grado de informatividad que presenta.

También es clave el aporte de Raúl Vera (2009) en su artículo «Los siete criterios de calidad de un texto», donde se establecen algunos parámetros que permiten la toma de decisiones sobre qué aspectos de la estructura deben ser editados o corregidos con profundidad. Según propone Vera, se trata de un conjunto de criterios de recta escritura que, aplicados a un texto, aseguran un estándar de calidad formal tanto en la escritura como en la fluidez de la lectura (visual silenciosa u ortolográfica).

El primero de los criterios se basa en la ortolectografía, es decir, la capacidad operativa para leer o escribir correctamente un texto, la obtención de un texto limpio de errores caligráficos o tipográficos. Cuando se falla en este aspecto, si el lector tiene la voluntad de acercarse a una obra con estas características, necesariamente ralentiza la lectura porque debe reponer, corregir o sobreinterpretar el contenido del texto.

El segundo se basa en la ortología, aquella parte de la gramática que enseña a pronunciar correctamente las palabras. Es el arte de hablar con recta dicción. En cuanto a las manifestaciones escritas, la ortología se concentra en cuestiones puramente ortográficas que pueden entorpecer la lectura, como la falta de tildes, las metátesis, el uso de una consonante por otra, etcétera.

El tercero, en la ortosintaxis, es decir, en la correcta construcción estructural de enunciados y de oraciones que forman los textos escritos. Se asocian con la ortosintaxis las reglas de puntuación de enunciados y de oraciones, los vicios de «arquitectura» de 
una oración, como la intercalación excesiva de cláusulas, los problemas de concordancia, los queísmos y los dequeísmos, etcétera.

El cuarto, en la ortosemántica, relacionada exclusivamente con el significado de un texto desde variados puntos de observación. Este criterio se construye sobre la base de las interpretaciones significativas y lógicas, la consistencia argumentativa del escrito, la precisión de los términos que se utilizan y la brevedad (la aplicación de las palabras justas).

El quinto, en la ortorredacción, la labor de poner por escrito todos los criterios de rectitud nombrados anteriormente. Pero, además, se focaliza en la fluidez estructural de la composición; en otras palabras, en la adecuación a un estilo de escritura de acuerdo con el género al cual un texto puede circunscribirse.

El sexto, en la ortopresentación, es decir, la capacidad de presentar un texto de manera agradable, capaz de atrapar la curiosidad natural de todo lector y de mantenerlo interesado en su contenido. Si bien este aspecto suele ser motivo de reflexión por parte del editor o del diagramador del contenido, es importante que el corrector tome conciencia de su importancia.

El séptimo y último criterio se basa en la ortointegridad como aplicación del sentido de completitud de un texto. Se trata de la habilidad de ser sistemático, no dejar ningún cabo suelto o algún aspecto sin desarrollar de acuerdo con lo prometido en el escrito.

Como señala Gómez Font (2017: 18), cualquier texto puede ser mejorado, en el sentido de que acerque la información de una manera más efectiva: «No basta con conocer las reglas, sino que también hay que poseer la sensibilidad necesaria para lograr que un texto se lea más cómodamente y para que sea — por qué no- más bello». Más aún, de lo dicho se deduce que no existe un solo tipo de corrector, sino que, de acuerdo con las características del texto, hoy se puede establecer una tipología profesional de acuerdo con los tipos textuales, los saberes que se requieren para intervenir el texto sin cambiar su esencia y el soporte en que se publica

\section{Una reflexión final}

La percepción general sobre las tareas de los correctores está sesgada por la sombra de las malas experiencias de quienes alguna vez publicaron. Desde entonces, el oficio de corrector ha suscitado múltiples polémicas.

Un corrector de textos es una persona por lo general formada, ya sea académicamente o por el curso de la experiencia, que asume el papel de primer lector o de lector mediador, con la intención de revisar si, en los textos que se le presentan, existen errores y, de haberlos, buscar la mejor forma de enmendarlos. Muchas veces, esta tarea se desarrolla en diálogo con el autor; otras tantas, se toman las decisiones junto con el editor; pero, en la mayoría de los casos, el corrector se encuentra solo y debe asumir la responsabilidad de elegir un criterio consistente con el tipo textual al que responda el escrito que debe corregir y llevar a cabo la detersión del texto de acuerdo con las normas lingüísticas correspondientes.

Para muchos, la corrección es una forma de vida. Los profesionales que se dedican a esta tarea cumplen la misión de facilitar la manera en que se comunican las personas 
y permiten enriquecer el vínculo entre ellas a través de palabras claras. Así como Quintiliano sostenía que la retórica presentaba una dimensión ética, en la corrección, la idea de bien marca el rumbo de la tarea.

A lo largo del presente artículo se intentó describir la problemática y la importancia de desarrollar una teoría lingüística sobre la corrección que permita brindar algún tipo de base teórica al profesional que se encuentra ante un texto y debe establecer un criterio sólido para su accionar. Es necesario reivindicar la figura de los correctores, y, para ello, es necesario comprender que la correctología es una disciplina que se enmarca dentro de los estudios del lenguaje, se sustenta en teorías lingüísticas y merece el respeto académico correspondiente.

De lograrlo, se pondrá un poco de luz sobre una práctica que impulsa a los correctores actuales a investigar, al encuentro donde se puedan compartir puntos de vista y se reflexione sobre la identidad misma de la corrección: un oficio, una profesión, una disciplina o un arte.

\section{Referencias bibliográficas}

BENÍTEZ, M. (1925). Recuerdos de un corrector de imprenta. Buenos Aires: Ferrari.

BERNÁRDEZ, E. (1982). Introducción a la lingüística textual. Madrid: Espasa Universitaria.

BEAUGRANDE, R. DE; DRESSLER, W. (1997). Introducción a la lingüística del texto. Barcelona: Ariel.

CURIA, B.; H. MOLINA (2010). Los epígrafes en La cautiva. Alicante: Biblioteca Virtual Miguel de Cervantes. Disponible en: https://goo.gl/CjXUo4.

ECHEVERRÍA, E. ([1837] 2010). La cautiva. Alicante: Biblioteca Virtual Miguel de Cervantes. Disponible en: https://goo.gl/Xx4ZdZ.

ECO, U. (1979). El arte de la edición. s. d. e.

ESCANDELL VIDAL, M. V. (1996). Introducción a la pragmática. Madrid: Anthropos.

JAKOBSON, R. (1963). Essais de linguistique genérale. París: Minuit.

JOHNSON-LAIRD, P. (1983). Mental Models. Towards a Cognitive Science of Language, Inference, and Consciousness. Cambridge: Harvard University.

GÓMEZ BELART, N. (2019). La corrección de textos. Un estudio funcional del vínculo entre el autor y el corrector. Tesis doctoral.

GÓMEZ FONT, A. (2017). Prólogo. En Rodríguez, S., Manual de corrección de textos. Técnicas, consejos y apuntes de clases. Lima: Escuela de Edición de Lima, pp. 17-20.

KERBRAT-ORECCHIONI, C. (1990). Les interactions verbales. París: Armand Colin.

LAGOMARSINO, R. E. (1957). Savia y follaje del libro. Buenos Aires: Sudamericana. LOTMANN, J. M. (1972). Vorlesungen zu einer strukturalen Poetik. Munich: Fink. LOTMANN, J. M. (1978). Estructura del texto artístico. Madrid: Istmo.

MELÚS, P. [con la cooperación de Francisco Millá] (1937). El libro del corrector. Vademécum de los escritores y de los profesionales de la tipografía. Barcelona: Neotipia.

MIRAGAYA, E. (1945). Diccionario de correcciones y palabras que se emplean equivocadamente en sustitución de las verdaderas. Buenos Aires: Ebro. 
PELLEGRINI, C. (1948). La industria gráfica argentina. Buenos Aires: Universidad de Buenos Aires. Facultad de Ciencias Económicas. Biblioteca «Alfredo L. Palacios».

RAFOLS, J. M. (1947). Acento impreso. Notas para correctores. Buenos Aires: Arbó.

REYES, G. (1990). La pragmática lingüística. Barcelona: Montesinos.

ROSENBLAT, A. (1967). El criterio de corrección lingüística: Unidad o pluralidad de normas en el castellano de España y América. Separata.

SPERBER, D.; WILSON, D. (1986). Relevance. Communication and Cognition. Oxford: Blackwell.

SWALES, J. M. (1990). Genre Analysis. English in academic and research settings. Cambridge: Cambridge University Press.

VAN DIJK, T. (1980). La ciencia del texto. Barcelona/ Buenos Aires: Paidós.

VAN DIJK, T. (2006). De la gramática del texto al análisis crítico del discurso. Una breve autobiografía académica. Barcelona: Universidad Pompeu Fabra.

VAN DIJK, T.; KINTSCH, W. (1983). Strategies of Discourse Comprehension. Nueva York: Academic Press.

VERA, R. (2009). Los siete criterios de calidad de un texto. [s. d. l.]. Separata.

ZORRILLA, A. M. (2011). Una carrera para formar correctores de textos en lengua española. Primer Congreso Internacional de Correctores de Textos en Lengua Española, Buenos Aires, 14-16 de septiembre de 2011. Separata. 